\title{
Manufacturing of Microfluidic Sensors Utilizing 3D Printing Technologies: A Production System
}

\author{
Danial Khorsandi, ${ }^{1}$ Mehrab Nodehi, ${ }^{2}$ Tayyab Waqar ${ }^{(D)}{ }^{3,4,5}$ Majid Shabani, ${ }^{6,7}$ \\ Behnam Kamare, ${ }^{6,7}$ Ehsan Nazarzadeh Zare, ${ }^{8}$ Sezgin Ersoy ${ }^{(1 D},{ }^{3,4}$ Mohsen Annabestani, ${ }^{6}$ \\ Mehmet Fatih Çelebi, ${ }^{4}$ and Abdullah Kafadenk ${ }^{9}$ \\ ${ }^{1}$ Department of Biotechnology-Biomedicine, University of Barcelona, Barcelona 08028, Spain \\ ${ }^{2}$ Ingram School of Engineering, Texas State University, San Marcos, TX 78666, USA \\ ${ }^{3}$ The Institute of Pure and Applied Sciences, Marmara University, Istanbul 34722, Turkey \\ ${ }^{4}$ Department of Mechatronic Engineering, Technology Faculty, Marmara University, Istanbul 34722, Turkey \\ ${ }^{5}$ Arcelik A.S., 34950 Istanbul, Turkey \\ ${ }^{6}$ Istituto Italiano di Tecnologia, Centre for Materials Interfaces, Viale Rinaldo Piaggio 34, 56025 Pontedera, Pisa, Italy \\ ${ }^{7}$ The BioRobotics Institute, Scuola Superiore Sant'Anna, Viale Rinaldo Piaggio 34, 56025 Pontedera, Pisa, Italy \\ ${ }^{8}$ School of Chemistry, Damghan University, Damghan 36716-41167, Iran \\ ${ }^{9}$ UNAM-National Nanotechnology Research Center and Institute of Materials Science and Nanotechnology, \\ Bilkent University, Turkey
}

Correspondence should be addressed to Sezgin Ersoy; sersoy@marmara.edu.tr

Received 20 January 2021; Accepted 25 July 2021; Published 11 August 2021

Academic Editor: Jianbo Yin

Copyright (c) 2021 Danial Khorsandi et al. This is an open access article distributed under the Creative Commons Attribution License, which permits unrestricted use, distribution, and reproduction in any medium, provided the original work is properly cited.

\begin{abstract}
3D integrated microfluid devices are a group of engineered microelectromechanical systems (MEMS) whereby the feature size and operating range of the components are on a microscale. These devices or systems have the ability to detect, control, activate, and create macroscale effects. On this basis, microfluidic chips are systems that enable microliters and smaller volumes of fluids to be controlled and moved within microscale-sized (one-millionth of a meter) channels. While this small scale can be compared to microfluid chips of larger applications, such as pipes or plumbing practices, their small size is commonly useful in controlling and monitoring the flow of fluid. Through such applications, microfluidic chip technology has become a popular tool for analysis in biochemistry and bioengineering with their most recent uses for artificial organ production. For this purpose, microfluidic chips can be instantly controlled by the human body, such as pulse, blood flow, blood pressure, and transmitting data such as location and the programmed agents. Despite its vast uses, the production of microfluidic chips has been mostly dependent upon conventional practices that are costly and often time consuming. More recently, however, 3D printing technology has been incorporated in rapidly prototyping microfluid chips at microscale for major uses. This state-of-the-art review highlights the recent advancements in the field of $3 \mathrm{D}$ printing technology for the rapid fabrication, and therefore mass production, of the microfluid chips.
\end{abstract}

\section{Introduction}

3D integrated microfluidic systems are engineered devices that actualize the precise routing of microsized fluidic streams for specific physicochemical and biological applications $[1,2]$. Due to such precision, the use of microfluidic devices is commonly known to be able to reduce the con- sumption of materials and regulate fluid flow in essential microscale environments [3]. The development of microfluid systems took place during the 1970s and has found numerous applications in automobile industry [4-7], medical technology [8-10], printing [11, 12], and sensor systems [13-15], as well as optical devices [16-18]. Despite such vast applications, in general, MEMS products are most commonly 
manufactured using traditional methods which include surface microprocessing [19], body microprocessing [20], and LIGA (Lithographie, Galvanoformung, Abformung (Lithography, Electroplating, and Molding)) [21]. These methods provide MEMS products to be more detailed and clearer by increasing the number of manufacturing steps and processing units that, in turn, increases the overall costs associated with the production of MEMS. In the same way, for small lab-scale research, this process generally results in a long and costly cycle which is not favoured. To address this, most recently, additive manufacturing (3D printing) techniques have been introduced which can potentially eliminate the disadvantages of conventional production methods used in microfluidic fabrication. Such $3 \mathrm{D}$ products can be used as microfluidics [22-24], micromechanical systems [25, 26], optical systems [27], cell structures [28], and biomedical devices [28-31].

With the term 3D printing being commonly defined as "a process of joining materials to make objects from 3D model data, usually layer upon layer, as opposed to subtractive manufacturing methodologies" 32 , 3D printing, in that respect, can potentially actualize rapid and easy prototyping and increase the quality of the produced microfluidic devices using different fabricating techniques. Such techniques to produce microfluidic chips include but are not limited to inject 3D printing, stereolithography (SL), fused deposition modeling (FDM), material jetting, binder jetting, directed energy deposition, and vat photopolymerization [32, 33].

In addition to the type of printing technique used, the possibility of printing materials with high details using different printing filaments is another feature of 3D-printed microfluidic devices. The high transparency, as a result of utilizing transparent filament, can effectively allow careful monitoring of the fluid flow $[1,32,34]$. As a result, depending upon the designated uses, resolution, colour, printing dimensions, and proper filament can, therefore, be utilized.

This review presents the wide range of $3 \mathrm{D}$ printing technology and their application towards the fabrication of microfluidic sensors and systems. Introduction regarding the microfluidic chip technology, along with clean room, is provided at the beginning which is followed by the implementation of the state-of-the-art 3D printing technology and their applications for the fabrications of such systems.

\section{Microfluidic-Chip Technology}

Microfluidic chips can be defined as a collection of microchannels fabricated or constructed into a material, such as silicon, glass, and polymer. They contain a mixture of interconnected microchannels, which forms the microfluidic chip, to perform the target operation or detection. These microchannels are interfaced to the outside world through the chip, and it is via these holes that gases or fluids are introduced and removed from the microchip. Figure 1 shows the technological advancements that have been achieved right from the invention of photolithography to the lab on chip [35].

From Figure 1, it can be deducted that the developments that have been made in the field of microfluidic technology are derived from the advancements in integrated circuits
(IC) and photolithography [36]. The development of microflow sensors, micropumps, and microvalves dominates the early phase of microfluids [37-39].

An example of such implementation of microfluidic chip has been presented in the form of a $\mathrm{T}$-junction which is used to measure pressure [40]. It has been found that for filtration purposes, the " $T$ " connections on microfluidics become blocked over time by fluids in droplet form thus causing numerous application issues such as unwanted pressure increase and variation in fluid velocity and acceleration, producing undesirable results. As viscosity of the given fluid varies, viscosity retention conditions in microfluidic channels take place. For this reason, clogging may be inevitable for microlevel channels. Figure 2 shows details regarding the increase in blockage situation in a microfluidic chip according to time. Based on the figure, the output over " $\mathrm{T}$ " suddenly drops to $8 \mu \mathrm{m}$ level. At $0.4 \mathrm{~ms}$, it starts to block in the form of droplets in the channel it comes from. Therefore, other devices or methods should be incorporated in order to resolve this issue. One way to address this tendency, for instance, is the use of Laplace sensors to solve the problem of droplet blocking at such return or connection points. As shown in Figure 2, a turn in the " $w$ " radius is put and thus, although the area used is upstream, no narrowing has occurred in the channels.

Although microfluidic chip technology is still in its infancy, viscosity and laminar flow are the most common issues associated with its applications. According to Sanli et al. [28], viscosity can be divided into Newtonian- and non-Newtonian-type fluids. With the former referring to a fluid whose viscosity does not change when a force is applied (e.g., water, gasoline, and alcohol), the latter refers to a varying viscosity state of fluid when the applied force is a determinant of the materials' viscosity (e.g., starch-water or ketchup). In the same way, the flow motion of this process can be classified as laminar and turbulent flow which refers to the regularity of flow of a fluid in a given medium.

Extending on the above application, a lab-on-a-chip microfluidic device has been presented by Chien and Parce [41]. An algorithm has been developed, to address the clogging issue, which works by calculating the pressures in the multiport flow control study for lab-on-a-chip microfluidic device. For this purpose, additional devices with an external pressure increasing multiport input have been incorporated. Through this process, a pressurized fluid such as the dye mixture and enzyme assay is often used along with a computercontrolled multichamber pressure/vacuum control unit with voltage and current input which has been built using the syringes used in the system (see Figure 3 ).

In this experiment, it has been revealed that the external pressure control and the predicted expectations are very close to each other. In this way, it was emphasized that a new way can be incorporated for biochemical experiments with the lab on a chip. It was further noted that the ability to control fluids flowing in microfluidics is always a leading feature. In addition, it was shown in that it is possible to direct many fluids within channels using a multiport system.

Many similar terms such as "Micro-fluids," "MEMSfluids" or "Bio-MEMS," and "microfluidics" have also emerged over time as the name of a new research discipline 


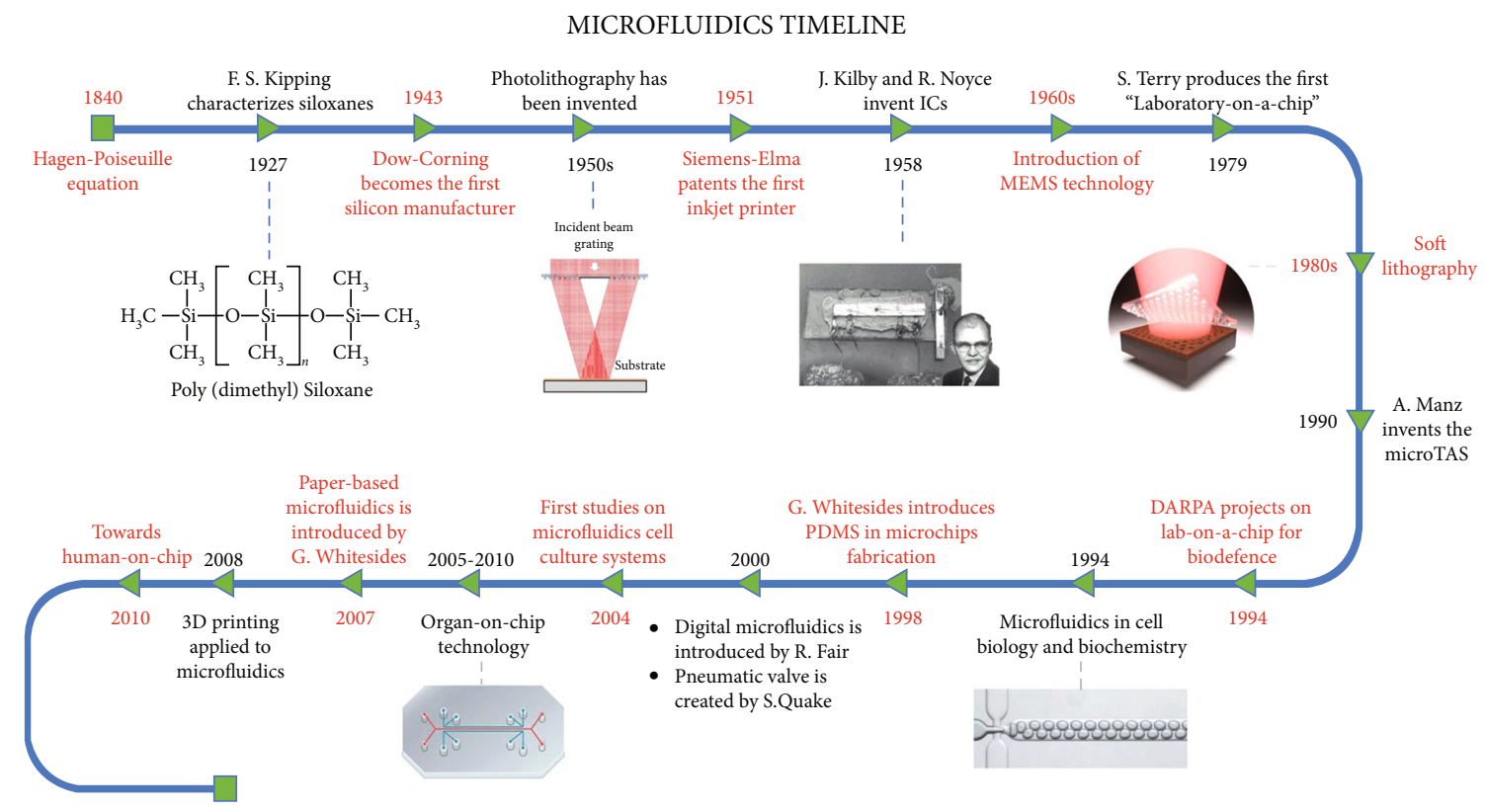

FIgURE 1: Timeline indicating advancements in the microfluidic technology [35].

dealing with transport paths and liquid-based devices at microscopic length scales. Figure 4 shows a scale to better distinguish microfluids or roughly MEMS and nanofluids. These devices can be completely distinguished from each other by size or volume [43-51].

Microfluidic devices have microchannels, having one dimension of at least 1 to $100 \mu \mathrm{m}$, that deal with the manipulation of liquids or gases $[53,54]$. The fabrication of these microchannels having such dimensions is due to the silicon microprocessing-enabled channels that allow the features to be produced with a precision of $1 \mu \mathrm{m}$. This technological achievement has enabled the reduction of micro- $\left(10^{-6}\right)$ litres to atto- $\left(10^{-18}\right)$ litres of liquid volume. For further illustration, the analytical and economic advantages of microfluidics are listed in Table 1.

2.1. Microelectromechanical Systems (MEMS). The term MEMS nowadays is used to refer to almost all types of miniaturised devices, such as 3D microstructures, mostly fabricated from silicon semiconductor while utilizing techniques that are the derivative of the recent developments in microfabrication industry [55]. With the development of semiconductor technologies, different MEMS production techniques have also started to develop. MEMS manufacturing technique procedures are somewhat similar as compared with ICs. With microfabrication, a large number of cheaper-cost products have been produced. Special microprocessing techniques have been developed for MEMS devices that cannot potentially be produced with traditional manufacturing techniques. These production techniques have advanced with the development of technology and can be classified as follows [56]:

(i) Bulk micromachining

(ii) Surface micromachining (iii) Wafer bonding

(iv) Lithographie, Galvanoformung, Abformung (LIGA)

(v) Other microprocessing techniques

The bulk micromachining technique is the oldest microprocessing technique. It is also highly preferred in MEMS technology. In this process, the material is fabricated within the desired limit via a number of steps. Bulk microprocessing is generally done in 2 different ways as wet and dry etching [57]. In wet etching, an acid-based liquid is applied on the specified material and is used which is widely used in MEMS production due to its fast and high selectivity. Nonetheless, the rate of etching can vary depending on many factors. Crystal structure of the material, residence time in the solution, type of doping, etc. are commonly known factors that affect this process [58]. A process which relies on the removal of the masked semiconductor material via the exposure to ion bombardment and reactive gases resulting in removing the masked portion of that material is known as dry etching [59]. These processes are further developed to make the final product, i.e., microfluidic chips, much smaller in size.

In addition, there are two types of wet chemical etching in the body microprocessing. These are classified as isotropic and anisotropic. In isotropic wet etching, since the rate of etching does not depend on the crystal structure of the material, the etching is distributed equally in all directions. It is made with chemicals such as hydrofluoric acid (HF) and hydrochloric acid $(\mathrm{HCl})$. Illustrated in Figure 5, abrasion proceeds at the same speed in all directions and circularshaped structures are obtained on the material. The anisotropic etching, however, does not spread equally in all directions since the rate of etching depends on the crystal structure of the material. It is made using solutions such as potassium hydroxide $(\mathrm{KOH})$ and tetramethylammonium hydroxide (TMAH). As can be seen in Figure 5, it carries 


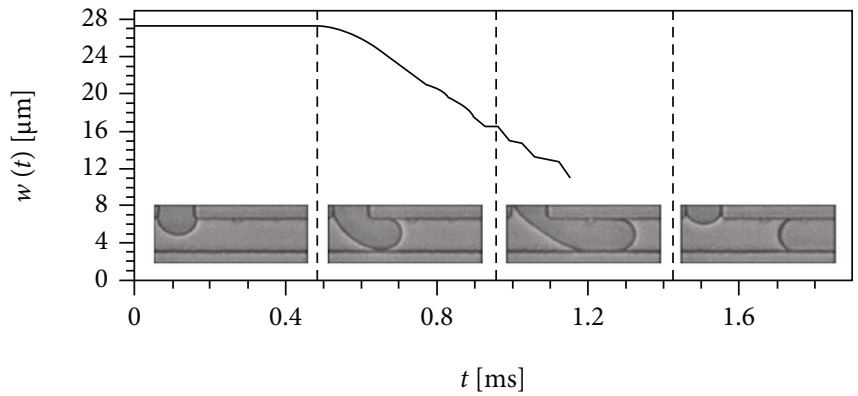

(a)

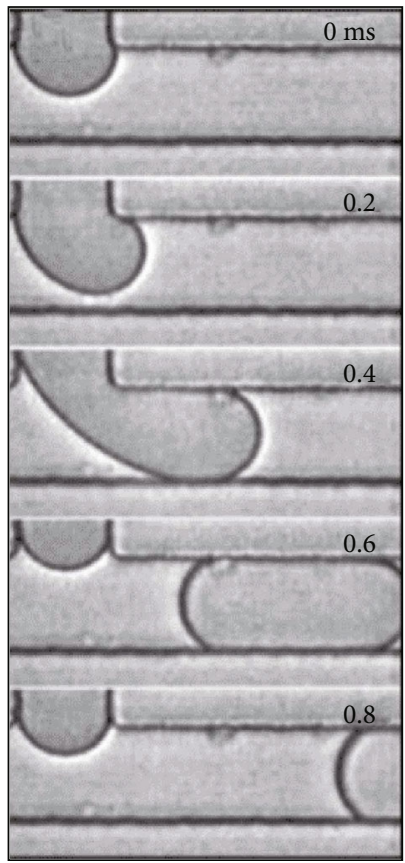

(c)

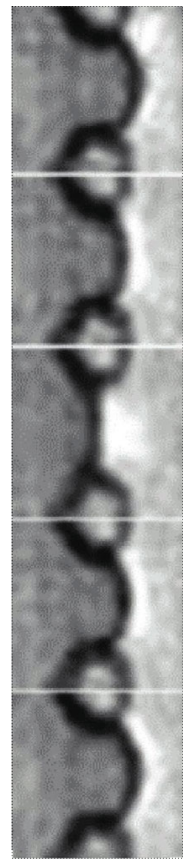

(d)

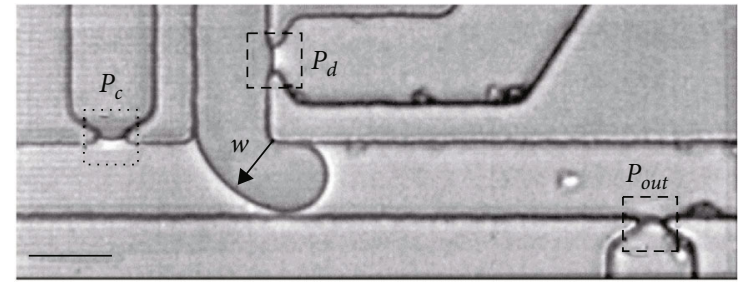

(b)

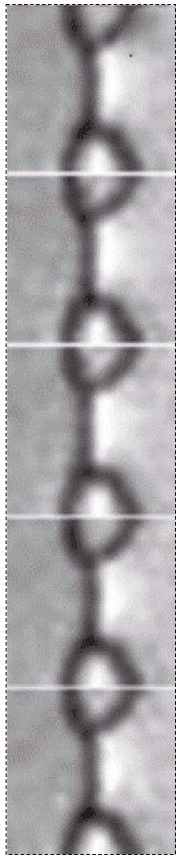

(e)

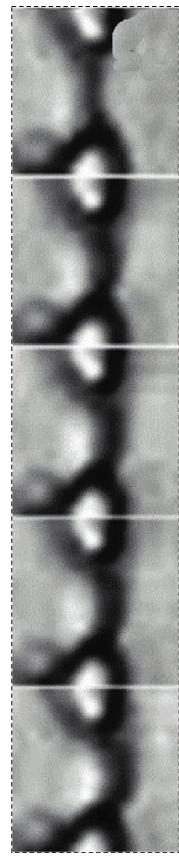

(f)

FIGURE 2: Microfluidic droplet: (a) time-dependent measurements of the emerging drop contour width at its narrowest point; (b) T-junction drop maker with three Laplace sensors used to measure the pressures in the continuous fluid upstream of the drop maker $P_{c}$, downstream of the drop maker $P_{\text {out }}$, and in the dispersed fluid $P_{\mathrm{d}} ;(\mathrm{c})$ image sequence showing the evolution of the formation of a drop; (d-f) simultaneous snapshots of the interfaces at the Laplace sensors at high magnification. Reprinted by permission from [40].

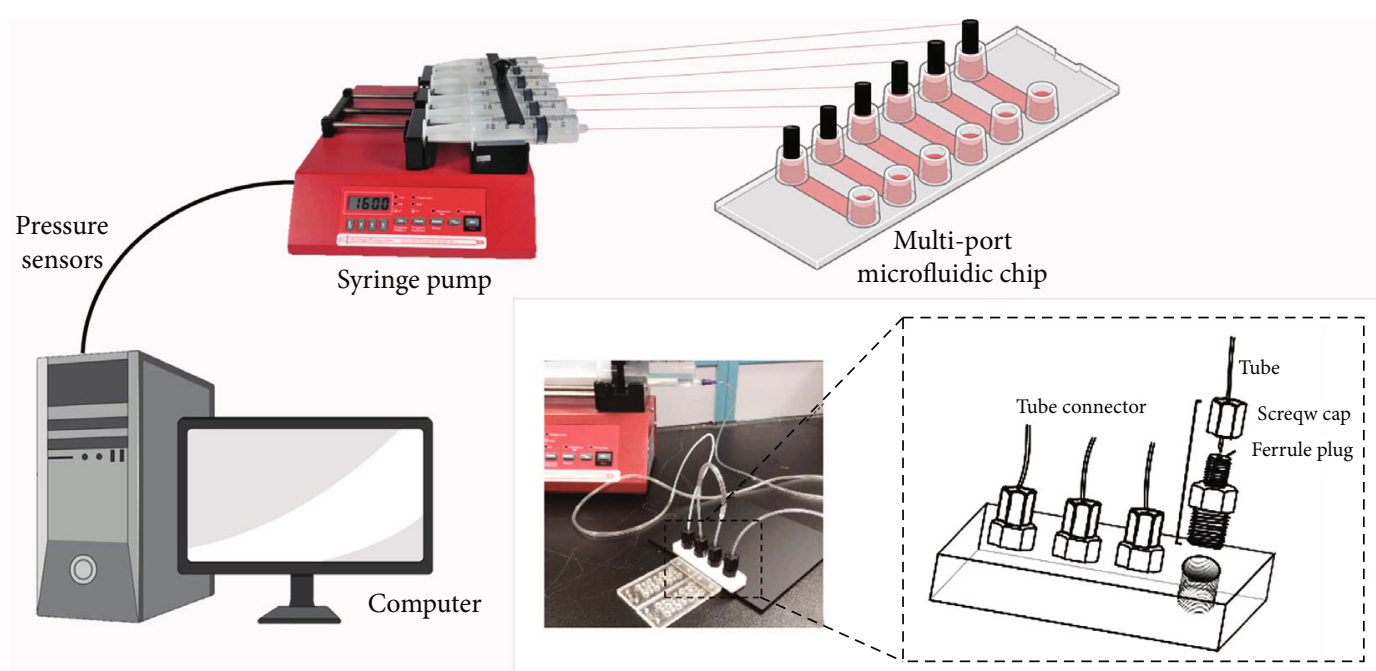

Figure 3: Multiport microfluidic connection diagram. Modified from [42]. 


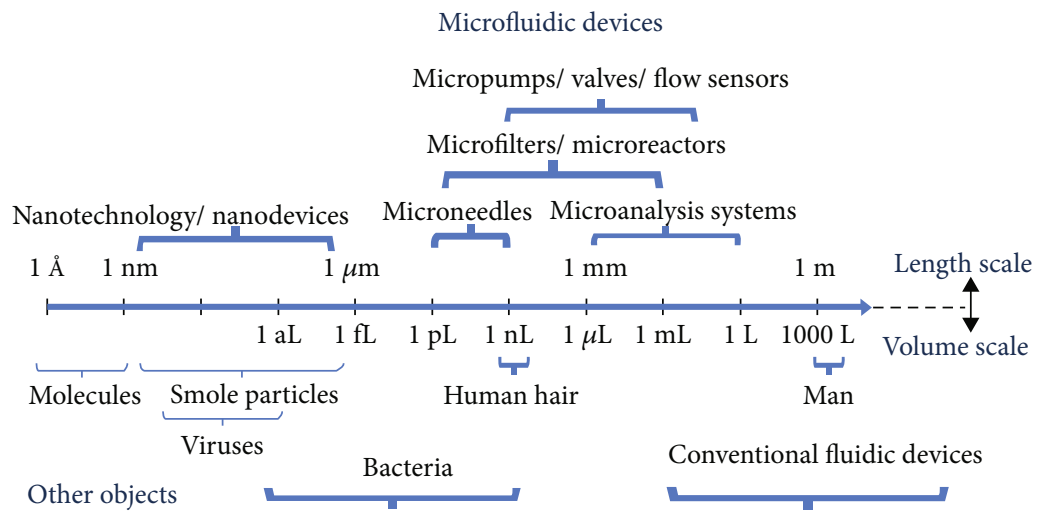

FIgURE 4: Some sample scales for comparison of microfluidic and nanofluidic device sizes [52].

TABLE 1: Advantages of microfluidics.

\begin{tabular}{|c|c|}
\hline Microfluid advantages & Description \\
\hline $\begin{array}{l}\text { Less sample and reagent } \\
\text { consumption }\end{array}$ & Microfluidic devices require less sample volume for traditional methods or analyses. \\
\hline Improved heat transfer & The higher surface area/volume ratio of microfluidic channels increases the effective thermal dispersion. \\
\hline Faster separations & Stronger electric fields cause faster sample throughput. \\
\hline Electrokinetic orientation & $\begin{array}{c}\text { Electroosmotic flow allows fluids to be pumped with a flat "plug-like" velocity profile applied only over } \\
\text { electric fields. }\end{array}$ \\
\hline Low power consumption & Fewer components and improved heat dissipation require less power input. \\
\hline Paralleling & Several assays can be mixed or analysed in parallel on a single chip. \\
\hline Portability & Thanks to system integration and low power consumption, it can perform portable conductive analysis. \\
\hline Improved separation efficiency & Efficiency in electrophoretic and chromatographic separations is proportional to $L / d$. \\
\hline
\end{tabular}

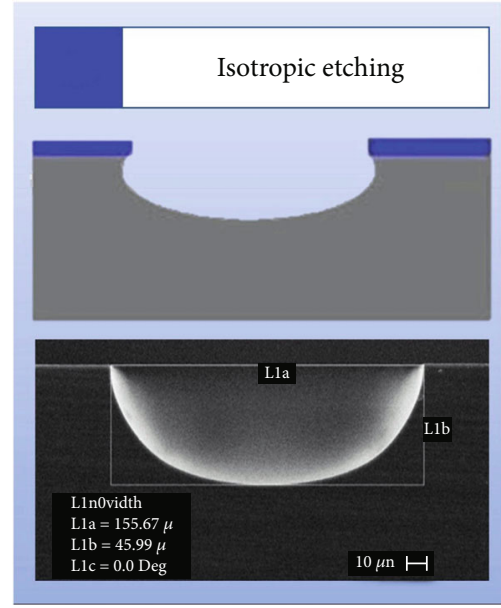

(a)

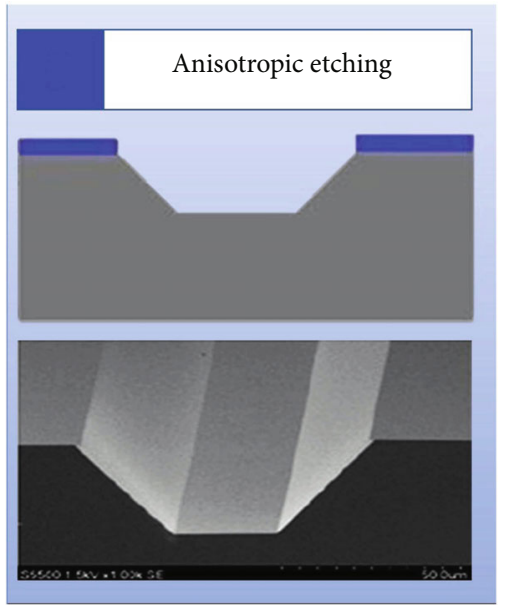

(b)

FIgURE 5: Isotropic (a) and anisotropic (b) etching. Reproduced with permission from [60, 61].

out different etchings in different crystal directions. The speed of erosion is also a variable with direct relation with the atomic density.

In general, however, any etching process requires a masking material that has a high selectivity with respect to the substrate material. Common masking materials for isotropic wet etching include silicon dioxide and silicon nitride. Silicon nitride has a lower wear rate compared to silicon dioxide and is therefore used more frequently [62].
The most commonly used method in the body microprocessing technique is deep reactive-ion etching (DRIE) with dry etching. DRIE is one of the new technologies of MEMS device manufacturing. With this technology, very high abrasion depth and height can be achieved. Assuming that the substrate plate used is silicon, the etching depth with this method can be in size of a thousand of microns [62].

Surface microprocessing is created by coating thin layers on the silicon plate. In body microprocessing, this is the 


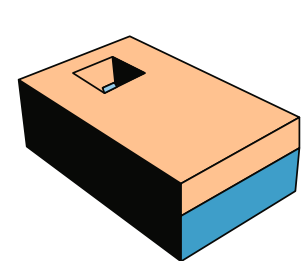

Deposit \& pattern oxide

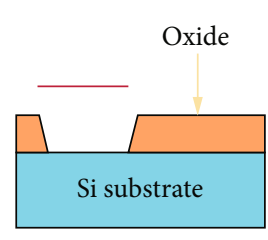

Sacrificial material: Silicon oxide

Structural material: Polycrystalline Si (poly-Si)

Isolating material (electrical/ thermal): Silicon nitride

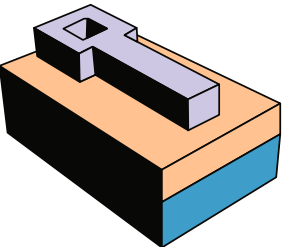

Deposit \& pattern poly
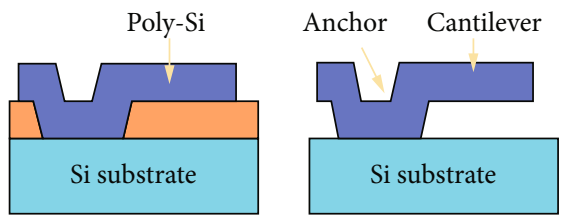

Sacrificial etch

Anchor Cantilever

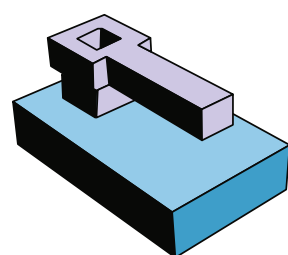

Figure 6: Microfluid surface microprocessing, reprinted with permission from [63]. opposite, and structures are created by material removal. As shown in Figure 5, a temporary coating (for example silicon dioxide) is first made on a plate. Then, the material of the final MEMS device to be produced is coated on this structure using a thin film. With the completion of these processes, the temporary material is removed with some solutions and the real material remains.

Examining the steps for the surface microprocessing, presented in Figure 6, it can be observed that the process is a bit complicated and likely to cause problems. Whether the properties of all materials and solutions to be used in this technique are suitable or not for this method should be compared in detail. One of the most important issues in this technique is that the solution to be applied to remove the temporary layer should be very careful against damage to the permanent material. It should be noted that etching processes cannot be too fast and effective at the same time.

The method of forming multiple layers at microlevels is commonly called the plate joining microprocessing technique (i.e., wafer bonding). There are three different types of microprocessing techniques used in this regard that include direct bonding, anodic bonding, and bonding using interlayer. In order for these layers to be combined, the layers must be smooth and clean [62].

Anodic coating, which is a special surface coating for aluminium, is applied as a sodium glass or silicone coating. This relationship is established by applying certain temperatures and tension. Metal, polymer, or glass plate is used for these intermediate plates. The direct joining method is usually achieved by adhering to the silicone layers $[64,65]$.

Lithography, Galvanoformung (electroplating), Abformung (also press molding) is a microprocessing technique consisting of words such as LIGA [66]. It is another technique that has emerged to respond to the demands and needs of the developing MEMS devices. It is the method that enables MEMS devices that cannot be produced with silicon processing techniques to be produced in $3 \mathrm{D}$-printed plastic structures, metal ceramics, etc. where LIGA technique is employed to reveal the structures.
Another micromachining technique, laser micromachining, works by applying energy to the required area by sending very short light through factors such as wavelength, energy, and power, depending on the laser type. Electrodischarge micromachining is another widely preferred microprocessing technique for materials with electrical conductivity. It is especially preferred in complex systems. Microprocessing with a focused ion beam is preferred for very small sizes (nanolevels). The main element of microprocessing, in this technique, is the voltage rise and fall. Voltages can range from a few kiloelectron volts $(\mathrm{keV})$ to a hundred times kiloelectron volts [62].

In general, microfluidic chips are devices that work on the principle of liquid transport, which significantly reduces the complexity and power consumption of mechanical tests. For example, electroosmosis is a process in which bulk electrolytic fluid in a channel is entrained through viscosity by moving ions near a naturally charged channel wall by the application of an electric field [67].

Several analytical performance measures can be improved through miniaturization. One of the most obvious advantages of the smaller channel sizes is reduced reagent consumption, resulting in less waste and more efficient testing. Reduced reagent consumption becomes particularly advantageous for many biological applications where reagents can be very expensive and sample volumes are often limited. In addition, the separation efficiency of chromatographic and electrophoretic systems is proportional to $\mathrm{L} / \mathrm{d}$ (the length of the separation channel over its diameter). Therefore, long and narrow channels provide improved peak-to-peak resolution.

Because they are very narrow, microfluidic channels also have flowed with very low Reynolds numbers; i.e., $\mathrm{Re}<1$ flow is laminar. Such laminar flows prevent additional dispersion from affecting the bandwidth of a separate plug. However, diffusion is more pronounced on smaller scales and is advantageous for mixing applications where mixing can only be done by diffusion, despite very laminar flow. Additionally, narrow channels dissipate heat more efficiently and allow 
stronger electric fields in electrophoretic systems without adverse heating effects on separation efficiency. As a result, tests will take less time as higher electric fields lead to faster separations.

2.2. Cleanroom System. The cleanroom system is a system used in production or scientific research and does not contain high levels of environmental pollutants. Some special measures should be taken in order to provide the desired level of cleanliness in the cleanroom system. Clean rooms are used in many sectors such as for the manufacturing of semiconductor devices, which is a key ingredient of MEMS [68].

The modern cleanroom system was invented by the American physicist Willis Whitfield. This work was conducted at the Sandia National Laboratory. Whitfield developed the cleanroom system in a modern way by using the filter system effectively in his study. Earlier cleanroom systems had many problems with unpredictable airflows [66, 68, 69].

Clean rooms can be used in any industrial area where small levels of particles can adversely affect the production process and can differ in size and complexity. Cleanroom systems are mainly used in the fields of biotechnology, semiconductor production, life sciences, pharmaceutical industry, and medical device production $[66,68,70]$.

Since cleanroom systems are used in many applications, there are many cleanroom classes according to their usage areas. The HVAC (Heating, Ventilating, and Air Conditioning) design that the cleanroom will use is also made accordingly. HVAC ventilation concerns many related areas.

Cleanroom systems are classified for ease of use. There are many classification standards available today. The most commonly used of these classification types is Federal Standard 209E and ISO 14644 Clean Room Standard that has been prepared for cleanroom systems and cleanroom environments [71].

As given in Table 2, for example, the habitable ambient air corresponding to the ISO 9 class contains a maximum of 35200000 particles with a diameter of $0.5 \mu \mathrm{m}$ and larger per cubic meter. An ISO 4 class cleanroom allows a maximum of only 352 particles per cubic meter of $0.5 \mu \mathrm{m}$ or greater. The actuator in this study was also produced in class 4 of ISO.

To control the fabrication processes, generally, validation procedures are carried out. Validation processes, in that sense, can be defined as control of systems and documentation. The main purpose of the validation process is to control every part of the fabrication process [71].

Cleanroom systems can withstand air, humidity, heat, and temperature controls and contain certain particles according to class types. Many tests are carried out in the cleanroom. The purpose of these tests is to understand that the system is working correctly. Otherwise, problems may arise according to the application to be made. The main tests performed are as follows:

(i) Measurements regarding the number of particles

(ii) Measurements related to pressure gauge operations (iii) Humidity and temperature treatments

(iv) Control tests

(v) Air control tests

(vi) Filter (Hepa) control tests [71]

2.3. MEMS Manufacturing Techniques with 3D Printer. Recently, with the development of MEMS production methods, 3D printers, which can manufacture materials through the additive manufacturing technique, have been a novel way for the production of these devices due to both cost-effectiveness and the ease in manufacturing. The ongoing requirements such as the basic workflow and clean room used in the production of MEMS make production processes difficult from time to time. However, by using 3D printing, MEMS can be produced in any potential environment with the additive manufacturing method (Figure 7).

Due to their rapid design iteration and because of their low production, infrastructure, and maintenance costs, 3D printing continues to be an encouraging alternative compared to traditional techniques such as lithography. The recent development in the technology allows the fabrication of complex microfluidic devices (Figure 8), makes procedures fasters, and is cheaper, therefore, making it attractive to more users.

With the recent advancements in $3 \mathrm{D}$ printing technologies, highly complex microfluidic devices can be fabricated via single-step, rapid, and cost-effective protocols, making microfluidics more accessible to users.

2.4. Photopolymerization 3D Printing (Also Stereolithography). Stereolithography refers to one of the earliest additive manufacturing technologies that emerged in the early 1980s, commonly used for creating models and prototypes through a selective curing of a photopolymer by a UV using laser. As a result, the word stereolithography, a combination of the words "stereo = solid" and "lithography = printing by light" is used for this process. After its introduction by $3 \mathrm{D}$ systems, other institutions produced microstereolithography system and were able to produce devices capable of 3D production at microlevels. At a microlevel, microstereolithography fabrication techniques used for MEMS fabrication are commonly examined in 3 groups:

(i) Projection microstereolithography (mask projection): projection microstereolithography $(\mathrm{P} \mu \mathrm{SL})$ is a multifunctional and low-cost process that enables rapid production of ceramic products by $3 \mathrm{D}$ microfabrication using complex microsized polymer structures, electrolysis, or resin additives [69]. $\mathrm{P} \mu \mathrm{SL}$ enables the rapid production of complex $3 \mathrm{D}$ microsized structures, on a layer-by-layer basis (see Figure 9(a)). In addition, $\mathrm{P} \mu \mathrm{SL}$ uses the most advanced digital microdisplay technology with patterns that are both digital and dynamic. This method combines the prominent features of common stereolithography and projection lithography, creating rapid photopolymerization of all structures with a microlevel layer resolution of UV light. The materials used during the manufacturing 
TABLE 2: Cleanroom standards (reproduced from ISO/DIS 14644-1) [72].

\begin{tabular}{|c|c|c|c|c|c|c|}
\hline \multirow[t]{2}{*}{ ISO classification number $(N)$} & \multicolumn{6}{|c|}{$\begin{array}{l}\text { Maximum allowable concentrations }\left(\text { particles } / \mathrm{m}^{3} \text { ) for particles equal to and greater than the }\right. \\
\text { considered sizes shown below }{ }^{\mathrm{a}}\end{array}$} \\
\hline & $0.1 \mu \mathrm{m}$ & $0.2 \mu \mathrm{m}$ & $0.3 \mu \mathrm{m}$ & $0.5 \mu \mathrm{m}$ & $1 \mu \mathrm{m}$ & $5 \mu \mathrm{m}$ \\
\hline ISO class 1 & $10^{\mathrm{b}}$ & $\mathrm{d}$ & $\mathrm{d}$ & $\mathrm{d}$ & $\mathrm{d}$ & $\mathrm{e}$ \\
\hline ISO class 2 & 100 & $24^{\mathrm{b}}$ & $10^{\mathrm{b}}$ & $\mathrm{d}$ & $\mathrm{d}$ & e \\
\hline ISO class 3 & 1000 & 237 & 102 & $35^{\mathrm{b}}$ & $\mathrm{d}$ & e \\
\hline ISO class 4 & 10000 & 2370 & 1020 & 352 & $83^{\mathrm{b}}$ & e \\
\hline ISO class 5 & 100000 & 23700 & 10200 & 3520 & 832 & $\mathrm{~d}, \mathrm{e}, \mathrm{f}$ \\
\hline ISO class 6 & 1000000 & 237000 & 102000 & 35200 & 8320 & 293 \\
\hline ISO class 7 & c & c & c & 352000 & 83200 & 2930 \\
\hline ISO class 8 & c & c & c & 3520000 & 832000 & 29300 \\
\hline ISO class $9^{g}$ & c & c & c & 35200000 & 8320000 & 293000 \\
\hline
\end{tabular}

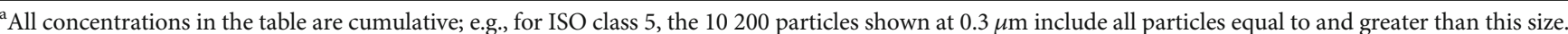
${ }^{b}$ These concentrations will lead to large air sample volumes for classification. Sequential sampling procedure may be applied. ${ }^{c}$ Concentration limits are not applicable in this region of the table due to very high particle concentration. ${ }^{\mathrm{d}}$ Sampling and statistical limitations for particles in low concentrations make classification inappropriate. ${ }^{\mathrm{e} S a m p l e}$ collection limitations for both particles in low concentrations and sizes greater than $1 \mu \mathrm{m}$ make classification at this particle size inappropriate, due to potential particle losses in the sampling system. ${ }^{\mathrm{f}}$ In order to specify this particle size in association with ISO class 5 , the macroparticle descriptor $M$ may be adapted and used in conjunction with at least one other particle size. ${ }^{8}$ This class is only applicable for the in-operation state.

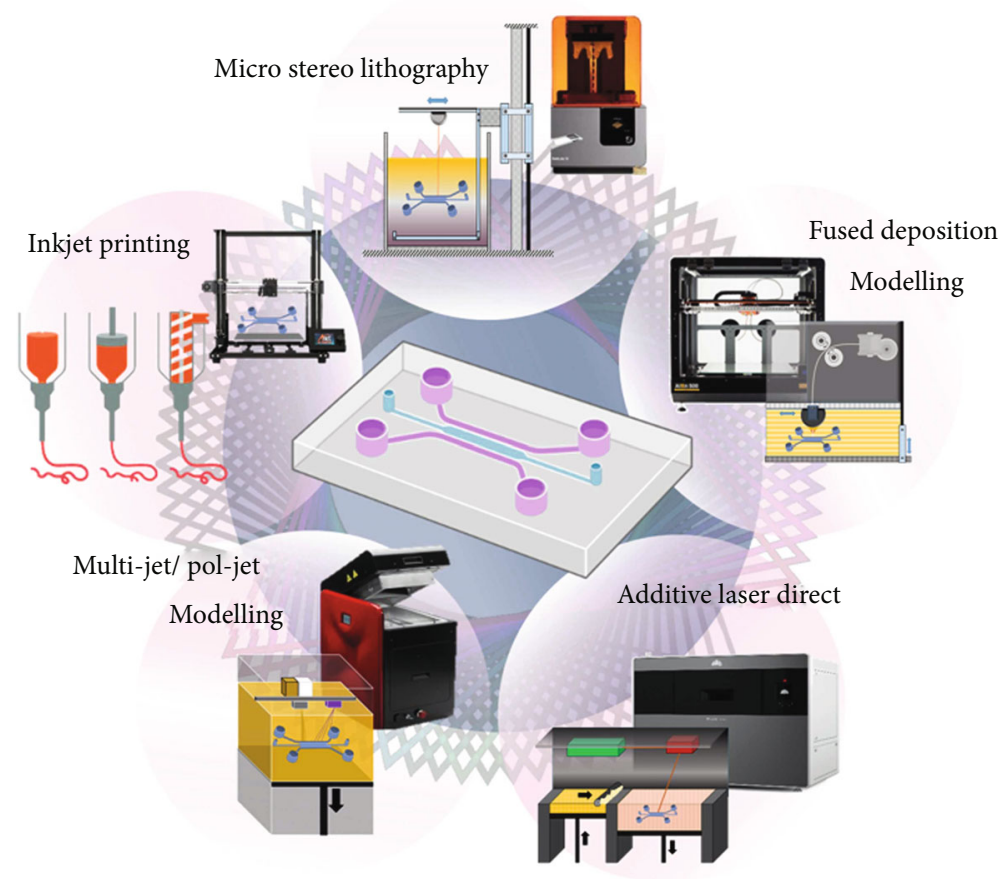

FIgURE 7: Additive manufacturing methods [73-75].

process can be changed easily [81]. The user can control the printing speed, UV light's intensity, and depth/height of the structure, letting them fabricate a variety of complex structures, such as spiral domes, pyramids, and microwells [82, 83] (Figure 9(b))

(ii) Two-photon polymerization: another $3 \mathrm{D}$ printing technique used is two-photon polymerization
(2PP) method which makes it possible to print out many complex products by making the fabrication production quickly and simply. Also, the high resolution in the printouts allows this technique to stand out [85]. In the 2PP method, it is the reactions that make it possible for the light-sensitive material to polymerize with a resolution of up to $100 \mathrm{~nm}$. Under these conditions, the material is only exposed to the laser beam for femtoseconds $\left(10^{-15}\right.$ seconds) [86] 


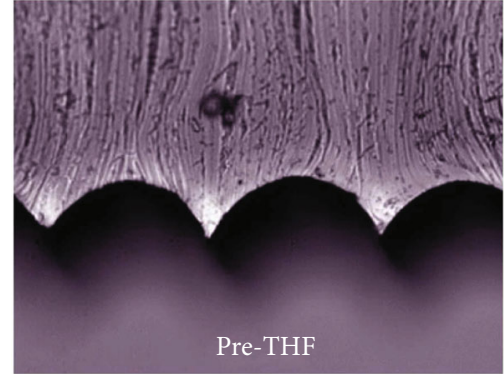

(a)

Cast PDMS and partially cure for $15 \mathrm{~min}$

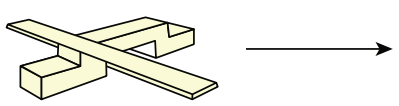

$3 \mathrm{D}$ printed master

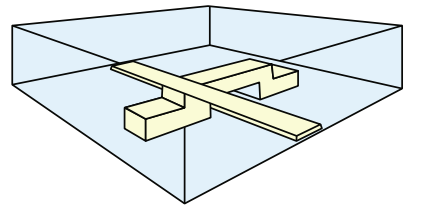

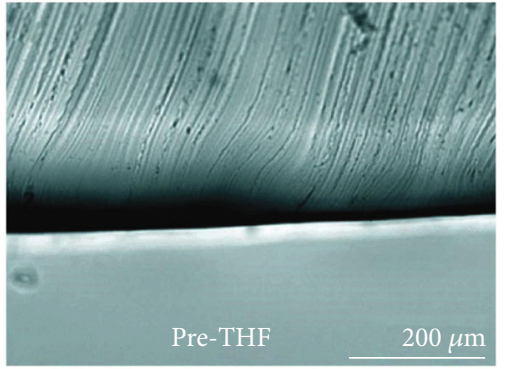

(b)

Peel off PDMS

from master

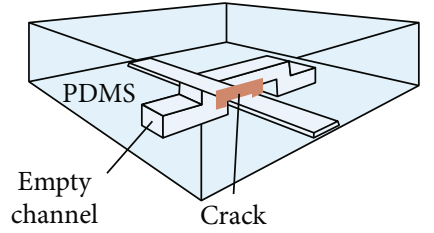

\section{Further cure the partially cured PDMS in the oven for $4 \mathrm{~h}$ at $80^{\circ} \mathrm{C}$ to thermally heal the cracks \\ 2. Thermally bond the chip on a slab of PDMS}

3. Loas dye solution
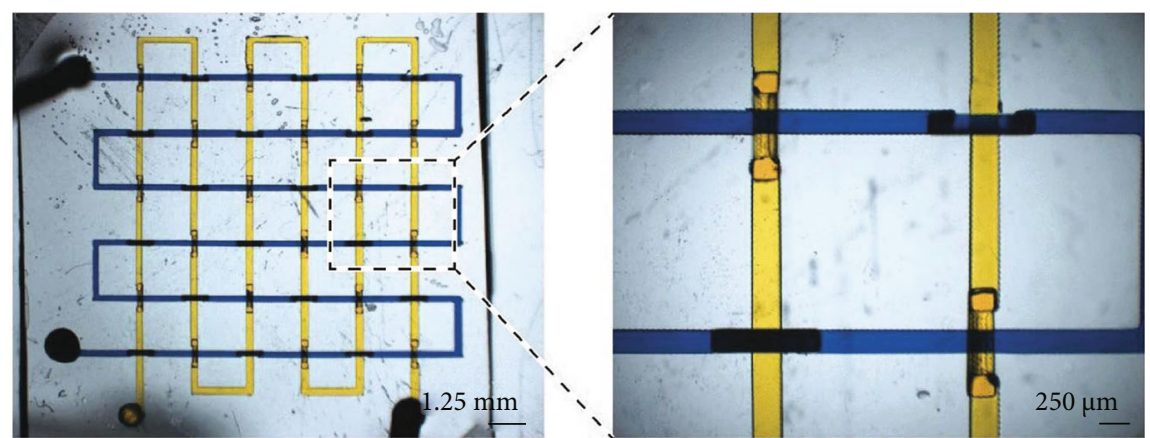

(c)

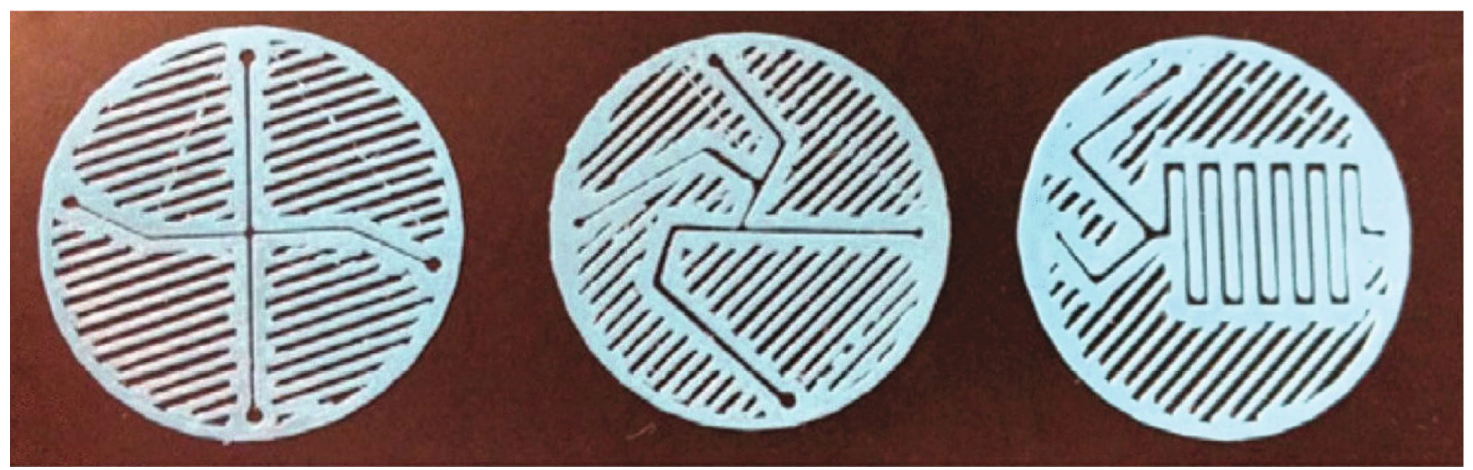

(d)

Figure 8: Template and surface of 3D-printed microfluidic devices. $(\mathrm{a}, \mathrm{b})$ Images showing the surface roughness of a polylactic acid fused deposition modeling (FDM) template before and after smoothing with tetrahydrofuran (THF) solvent. Panels adapted with permission from references [76, 77] (copyright 2016, Royal Society of Chemistry). (c) A method of casting a fully 3D device. Polydimethylsiloxane (PDMS) is cast over a 3D-printed template and allowed to partially cure. The PDMS is cracked and peeled off the template and then allowed to fully cure before filling with fluid for experiments. Adapted with permission from reference [78] (copyright 2015, Springer Nature). (d) Sandwich-style planar mixers are printed with FDM and then sandwiched between two surfaces with interface connections to form fluidic devices. Adapted with permission from reference [79] (copyright 2015, IOP Publishing) [80].

(iii) Continuous liquid phase production: in the continuous liquid phase production (CLIP) technique, product output can be obtained only with photopolymers in the material with microstereolithography. However, in $\mu \mathrm{SL}$, there is a long production time and a gradual interface between each printing layer. However, with the developing technology, the "continuous liquid phase production" defined as "CLIP" has completely eliminated the problems mentioned earlier. In this technique, microsized 3D 


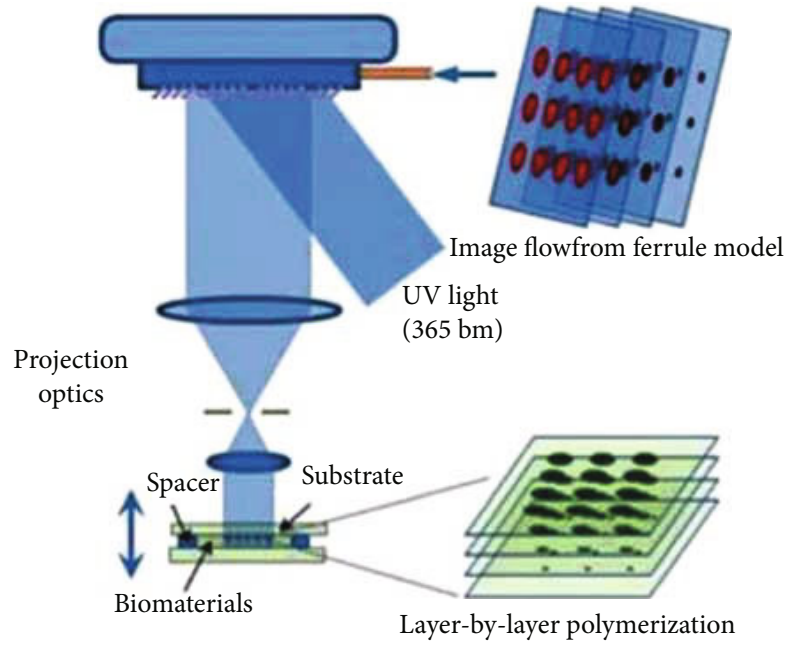

(a)

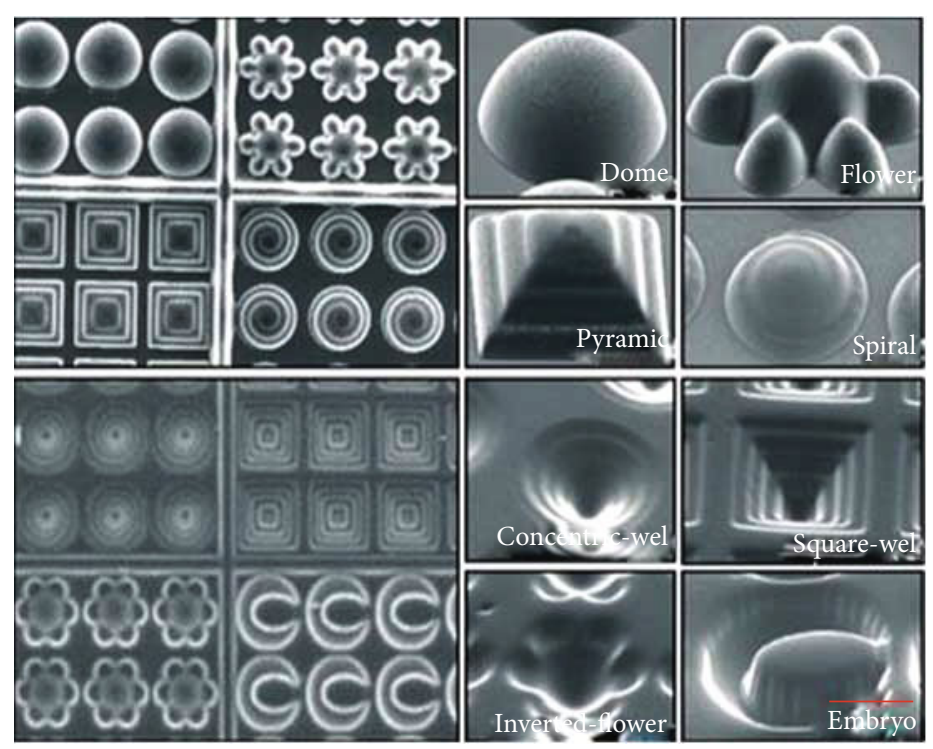

(b)

FIGURE 9: (a) Projection microstereolithography (P $\mu \mathrm{SL}$ ) setup: UV-light projects to the DMD mirror, which produces an optical pattern. The optical pattern projects across an optical lens to the photosensitive biomaterial to construct a 3D scaffold in a layer-by-layer method. (b) SEM images of the variety of printed microwells $[83,84]$.

mesoshapes without layers can be used at high speed and with vertical extrusion. The most striking feature of this technique is the provision of abundant oxygen permeability in terms of oxygen, which prevents photopolymerization. That is, in this technique, there is a continuous homogeneous production $[68,87]$. In addition, product outputs of elastic ceramics and biological materials can be obtained with this technology. Prototyping and mass production may also be preferred in large-width MEMS devices [68]

In general, 3D printing techniques are implemented to fabricate microfluidic channels that are designed in different shapes and sizes [22]. The designs are fabricated to analyse the performance of the $3 \mathrm{D}$ printer which was then found to be able to print structures with at least $50 \mu \mathrm{m}$ dimensions [22]. In this study, two different production methods were developed to optically improve the produced 3D structures. Those are the 3D structures being bonded to glass surfaces with polydimethylsiloxane (PDMS) and clear resin interlayers. The adhesion between the glass surface and the $3 \mathrm{D}$ structures was achieved with UV application for the resin and through the use of elevated temperature for PDMS. For different thicknesses of PDMS and resin interlayers, the bonding strength of the produced channels is commonly examined. Bright-field and fluorescence imaging properties of these channels are also usually analysed. As a result, twice the bond strength and comparable viewing capacity, compared to the PDMS-glass surface adhesion and compared to conventional plasma, can be achieved. In addition, using the presented production method, 3D structures are able to integrate protein-coated glass surfaces without disrupting the protein's functionality.
2.5. Powder Bed Fusion (Additive Laser Technique). Powder bed fusion refers to an additive manufacturing process in which a laser (thermal energy) selectively fuses regions of a powder bed. In this process, generally no support is required for the creation of the section. To produce microfluidic chips, different types of powder bed fusion are currently being used. Laser microsintering (LMS), for instance, is a powder metallurgy technique developed by Deckard and Beaman to make cast models from plastic powder. Its origin dates back to the 1980 s, and it is called selective laser sintering (SLS) $[88,89]$. In the working system, powder groups consisting of metal powders in a bed are melted or sintered by a laser beam. This process is a powder welding process to make a solid part or attachment to a previously determined computer-generated 3D model [90]. Most of the powders used in this technique are metals ( $\mathrm{Ag}, \mathrm{Al}, \mathrm{Cu}$, and stainless steels) or polymers in addition to some ceramics which can be processed using this technique [88].

Bohandy et al. [91] developed a matrix-assisted pulsed laser evaporation technique, which is a derivative of lasercontaining advanced transfer (LIFT), in 1986. This technique is used to create layers in one plane directly using the laser. It consists of a receiver and a transmitter. The donor material is transparent, and a thin-film method such as coating and spraying is used. In the data layer, it has two important features that can absorb the preferred wavelength and ensure a rapid supply of sufficient material in the case of continuous pressure $[92,93]$. Metals such as chromium, tungsten, gold, nickel, aluminium, copper, and vanadium, which are found in many different types, can be plated [94-96].

Laser chemical vapor coating (LCVD) is used in the production of complex parts, which can coat many different types of materials. The LCVD process is generally created in a vacuum chamber with the separation of the by-product 
(i) Print

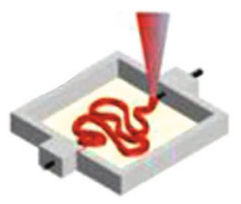

(i) Evacuate

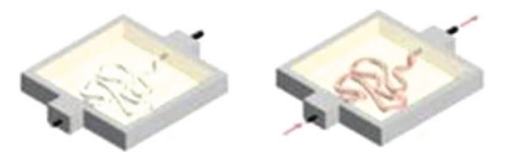

(a)

(ii) Cast

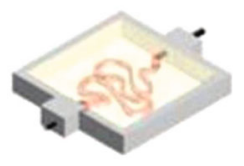

(ii) Add cells, perfuse

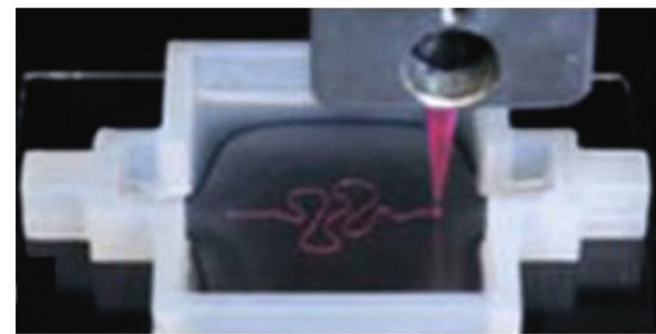

(b)

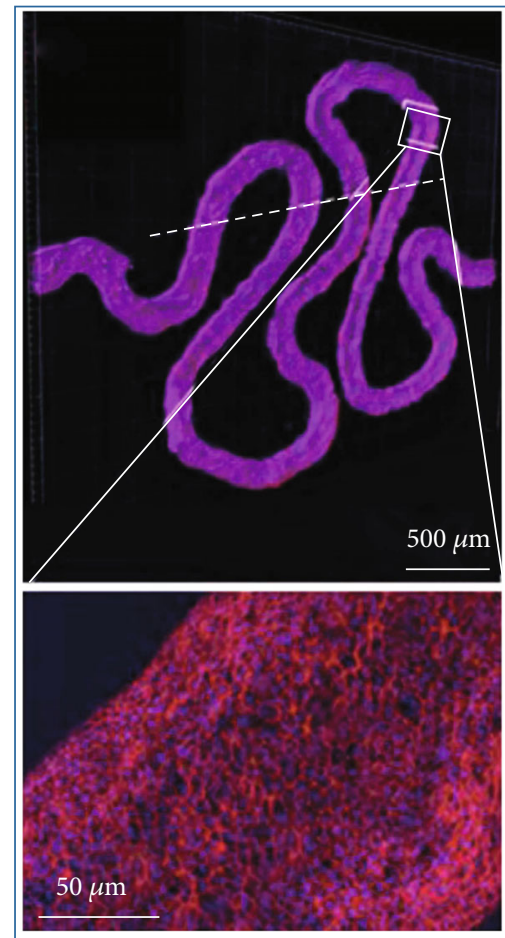

(c)

FIGURE 10: 3D-printed organs on chips in an ink-based printer technique: (a) illustration and (b) photograph of the extrusion-printing process of a kidney proximal tubule on a chip. (c) An immunofluorescent stained image of the inkjet-printed tubule [84].

that a precursor gas accumulates on the structure by laser beam during scanning. There are two laser-assisted groups that are divided into pyrolytic LCVD and photolytic LCVD. The process in which the precursor gas is thermally decomposed by laser heat on the layer is called the pyrolytic LCVD process while the process by which photon energy is absorbed by the precursor gas is called the photolytic LCVD process [97] which is commonly used for the metal deposition process, carbonyl, alkyl, halogen, oxyhalite, etc. while precursor gases are preferred; alkyls or alkyl halogens are generally preferred for semiconductors [98-102].

2.6. Material Jetting (Ink-Based 3D Printing and Extrusion). Extrusion-based and material jetting $3 \mathrm{D}$ printing refers to a series of $3 \mathrm{D}$ printers that utilize a nozzle to directly print molten materials from a constant cross-sectional diameter nozzle. In this process, the molten material is liquified and bonds with the previously printed layers and sections. In this process, photomonomers, in solution with nanoparticles in colloidal suspension, and inks, which can be found in several different types in organic or inorganic solvents, containing metallic and nonmetallic components are selectively deposited. This process can further be divided into the following [103]:

(i) Direct part printing: this includes printing sections with photopolymers and waxes by directly printing the parts 

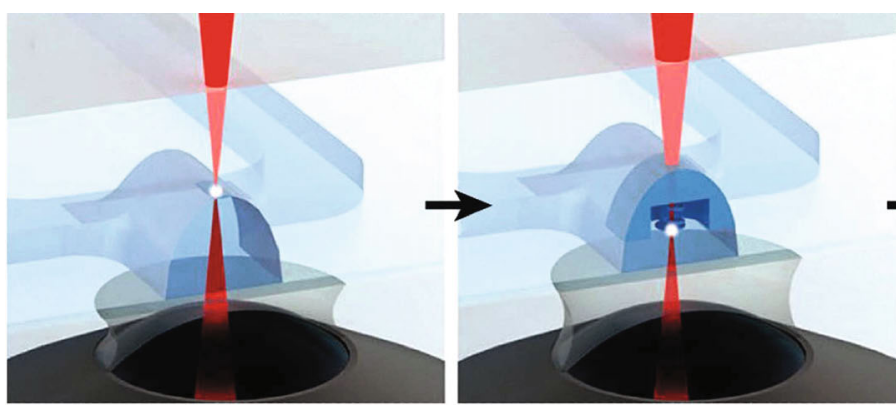

(a)

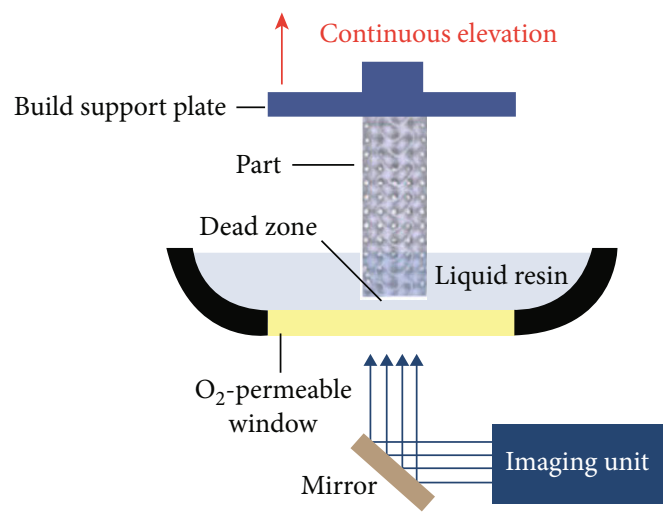

(b)

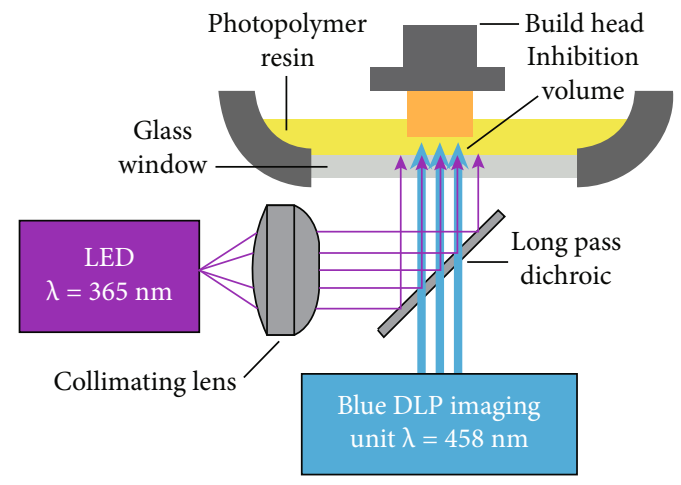

(c)

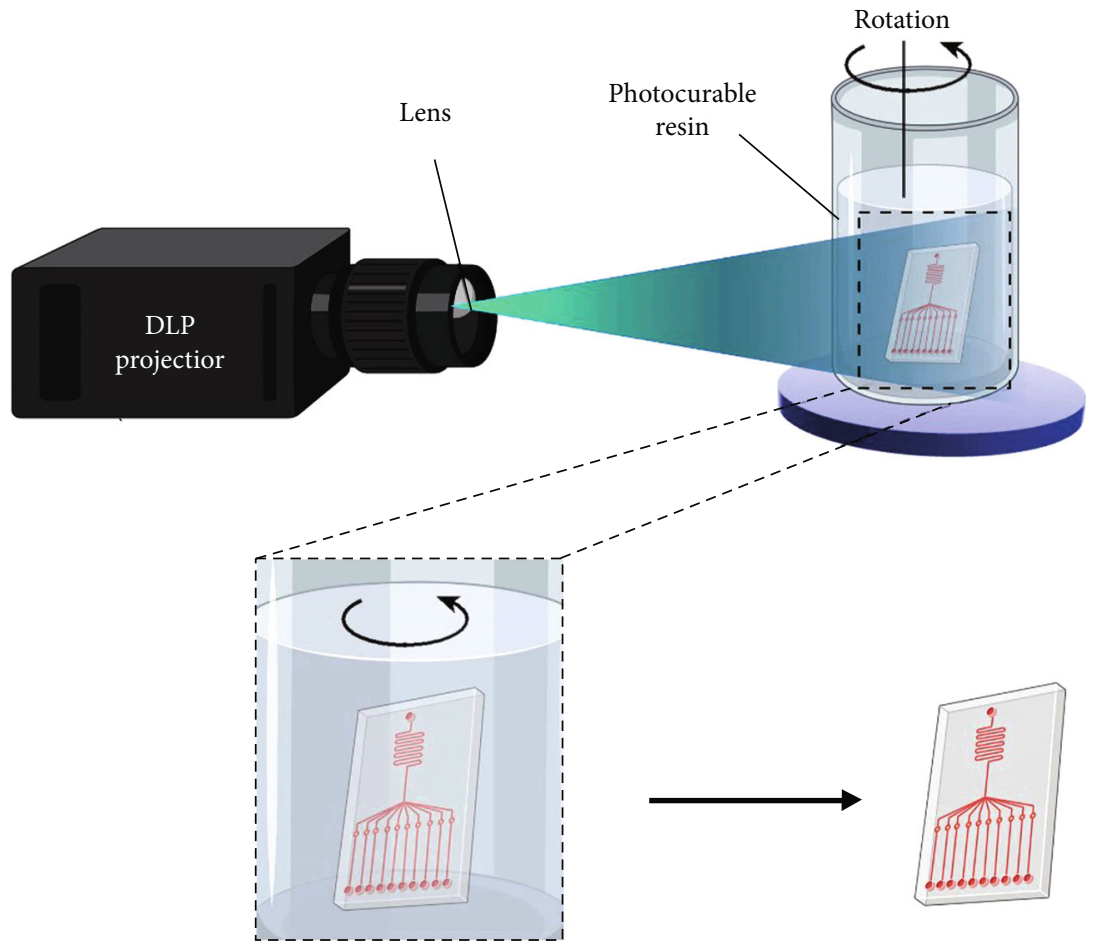

(d)

FIGURE 11: New approaches for 3D printing of microfluidic devices and structures. (a) Two-photon polymerization of a spring diode inside a microfluidic channel. Adapted with permission from reference [107]. (b) Instrumentation setup for CLIP. The build platform is continuously raised out of a resin vat, and polymerization is enabled by an oxygen-inhibited dead zone above a permeable window. Adapted from reference [76] (copyright 2015, AAAS). (c) Instrumental setup for an alternate approach to CLIP. Polymerization is initiated by blue light and inhibited by UV light. Adapted with permission from reference [77]. (d) Image angle breakdown and instrumental setup for CAL 3D printing. Abbreviations: CAL: computed axial lithography; CLIP: continuous liquid interface printing; DLP: digital light processing [79, 80]. 
(ii) Binder printing: this technique refers to a rather broad class of processes whereby the binder is printed onto powder bed

To manufacture microfluidic chips and devices by using this technique, generally the direct part printing is utilized.

An application of the ink-based printer technique is shown in Figures 10 and 11.

In this process, a thermal actuator or piezoelectric actuator is commonly used to allow ink droplets to contact a surface. In this section, ink droplets can be used as separate droplets from time to time (the drop-and-demand (DoD) method) or as a continuous inkjet (CIJ) method by recycling unused ink fluid. Since the control area for the material distributed in the DoD is larger, it is more suitable for microfabrication [104].

2.7. Other 3D Printing Techniques. Other 3D printing techniques include Multi Jet technology which combines inkjet and stereolithography 3D printing methods. A multi-inkbased model is created by spraying photopolymer resins on a layered structure. PolyJet materials harden as a result of being exposed to UV rays for a short time, and the product is formed after stratification. Multi-Jet technology enables the production of especially smoother, more precise, and higher resolution products.

In addition, hybrid products can be produced by mixing plastic, flexible, and transparent material derivatives in $3 \mathrm{D}$ printing $[105,106]$.

Nowadays, it is easy to print 3D products between 70 and 200 microns with Multi Jet and FDM technologies. This scale is not very suitable for MEMS devices.

\section{Conclusions and Future Perspective}

Microfluidic chips and devices are able to downscale biochemical and biomedical processes to a portable micro- and nanosized scale. Such engineered devices allow a better control over fluid flow and provide microenvironmental control over variables that can have major uses in autonomous applications. As a result of its numerous contributions, in this review study, the manufacturing technologies used in the design and manufacture of micro- and nanoscale sensors and their subcomponents were examined. According to the results of the experimental studies, the following inferences have been made:

(i) Options in production technologies include changes according to the working and measuring features of the designed sensor. There is an increasing tendency to use the FDM method in the selection of the production method. However, it is related to the properties desired to be produced by chemical or physical interaction

(ii) The sensors to be produced have morphological features that can be used in every field from biomedical to aviation. However, product expectations may not be sufficient for uniform material composition. Printers that are hybrid and can produce different types of materials (metal-ceramic-polymer) should be used in expanding the product range. The selection of production methods varies in the characteristics of the sensor to be produced

(iii) The choice of matrix material in the production of composite, coating, and other production methods causes all properties to change

\section{Conflicts of Interest}

The authors declare that there is no conflict of interest regarding the publication of this paper.

\section{References}

[1] G. Weisgrab, A. Ovsianikov, and P. F. Costa, "Functional 3D printing for microfluidic chips," Advanced Materials Technologies, vol. 4, no. 10, p. 1900275, 2019.

[2] J. Qiu, Q. Gao, H. Zhao, J. Fu, and Y. He, "Rapid customization of 3D integrated microfluidic chips via modular structure-based design," ACS Biomaterials Science \& Engineering, vol. 3, no. 10, pp. 2606-2616, 2017.

[3] A. K. Au, N. Bhattacharjee, L. F. Horowitz, T. C. Chang, and A. Folch, "3D-printed microfluidic automation," Lab on a Chip, vol. 15, no. 8, pp. 1934-1941, 2015.

[4] K. S. Teh, "Additive direct-write microfabrication for MEMS: a review," Frontiers of Mechanical Engineering, vol. 12, no. 4, pp. 490-509, 2017.

[5] C. Acar, A. R. Schofield, A. A. Trusov, L. E. Costlow, and A. M. Shkel, "Environmentally robust MEMS vibratory gyroscopes for automotive applications," IEEE Sensors Journal, vol. 9, no. 12, pp. 1895-1906, 2009.

[6] D. S. Eddy and D. R. Sparks, "Application of MEMS technology in automotive sensors and actuators," Proceedings of the IEEE, vol. 86, no. 8, pp. 1747-1755, 1998.

[7] B. P. Gogoi and D. Mladenovic, "Integration technology for MEMS automotive sensors," in IEEE 2002 28th Annual Conference of the Industrial Electronics Society. IECON 02, Seville, Spain, 2002.

[8] A. Ostendorf and B. N. Chichkov, "Two-photon polymerization: a new approach to micromachining," Photonics Spectra, vol. 40, no. 72-undefined, 2006.

[9] D. Panescu, "MEMS in medicine and biology," IEEE Engineering in Medicine and Biology Magazine, vol. 25, no. 5, pp. 19-28, 2006.

[10] D. L. Polla, A. G. Erdman, W. P. Robbins et al., "Microdevices in medicine," Annual Review of Biomedical Engineering, vol. 2, no. 1, pp. 551-576, 2000.

[11] A. I. Tsung Pan, Monolithic thermal ink jet printhead with integral nozzle and ink feed, 1987.

[12] K. Silverbrook, Ink jet print device and print head or print apparatus using the same, 2000.

[13] C. Hagleitner, A. Hierlemann, D. Lange et al., "Smart singlechip gas sensor microsystem," Nature, vol. 414, no. 6861, pp. 293-296, 2001.

[14] Q. Wan, Q. H. Li, Y. J. Chen et al., "Fabrication and ethanol sensing characteristics of $\mathrm{ZnO}$ nanowire gas sensors," Applied Physics Letters, vol. 84, no. 18, pp. 3654-3656, 2004.

[15] T. Waqar and S. Ersoy, "Design and analysis comparison of surface acoustic wave-based sensors for fabrication using 
additive manufacturing," Journal of Nanomaterials, vol. 2021, Article ID 5598347, 12 pages, 2021.

[16] Shi-Sheng Lee, Long-Sun Huang, Chang-Jin Kim, and M. C. $\mathrm{Wu}$, "Free-space fiber-optic switches based on MEMS vertical torsion mirrors," Journal of Lightwave Technology, vol. 17, no. 1, pp. 7-13, 1999.

[17] D. M. Marom, D. T. Neilson, D. S. Greywall et al., "Wavelength-selective $1 / \mathrm{spl}$ times/K switches using free-space optics and MEMS micromirrors: theory, design, and implementation," Journal of Lightwave Technology, vol. 23, no. 4, pp. 1620-1630, 2005.

[18] P. F. van Kessel, L. J. Hornbeck, R. E. Meier, and M. R. Douglass, "A MEMS-based projection display," Proceedings of the IEEE, vol. 86, no. 8, pp. 1687-1704, 1998.

[19] J. M. Bustillo, R. T. Howe, and R. S. Muller, "Surface micromachining for microelectromechanical systems," Proceedings of the IEEE, vol. 86, no. 8, pp. 1552-1574, 1998.

[20] M. Hoffmann and E. Voges, "Bulk silicon micromachining for MEMS in optical communication systems," Journal of Micromechanics and Microengineering, vol. 12, no. 4, pp. 349-360, 2002.

[21] J. Hormes, J. Göttert, K. Lian, Y. Desta, and L. Jian, “Materials for LiGA and LiGA-based microsystems," Nuclear Instruments and Methods in Physics Research Section B: Beam Interactions with Materials and Atoms, vol. 199, pp. 332-341, 2003.

[22] S. Keçili, Fabrication of microfluidic devices via 3D printer, 2019.

[23] Y. Li, Y. Fang, J. Wang et al., "Integrative optofluidic microcavity with tubular channels and coupled waveguides via two-photon polymerization," Lab on a Chip, vol. 16, no. 22, pp. 4406-4414, 2016.

[24] I. Unalli, S. Ersoy, and I. Ertugrul, "Microfluidics chip design analysis and control," Journal of Mechatronics and Artificial Intelligence in Engineering, vol. 1, no. 1, pp. 2-7, 2020.

[25] G. Nelson, R. A. Kirian, U. Weierstall et al., "Three-dimensional-printed gas dynamic virtual nozzles for x-ray laser sample delivery," Optics Express, vol. 24, no. 11, pp. 1151511530, 2016.

[26] I. Ertugrul and T. Waqar, "Fabrication of bidirectional electrothermal microactuator by two-photon polymerization," Current Nanoscience, vol. 16, 2020.

[27] C. Peters, M. Hoop, S. Pané, B. J. Nelson, and C. Hierold, "Degradable magnetic composites for minimally invasive interventions: device fabrication, targeted drug delivery, and cytotoxicity tests," Advanced Materials, vol. 28, no. 3, pp. 533-538, 2016.

[28] U. T. Sanli, H. Ceylan, I. Bykova et al., “3D nanoprinted plastic kinoform X-ray optics," Advanced Materials, vol. 30, no. 36, 2018.

[29] K. S. Worthington, L. A. Wiley, E. E. Kaalberg et al., "Twophoton polymerization for production of human iPSCderived retinal cell grafts," Acta Biomaterialia, vol. 55, pp. 385-395, 2017.

[30] C. A. Lissandrello, W. F. Gillis, J. Shen et al., "A micro-scale printable nanoclip for electrical stimulation and recording in small nerves," Journal of Neural Engineering, vol. 14, no. 3, p. 036006, 2017.

[31] M. Suzuki, T. Takahashi, and S. Aoyagi, "3D laser lithographic fabrication of hollow microneedle mimicking mos- quitos and its characterisation," International Journal of Nanotechnology, vol. 15, no. 1/2/3, p. 157, 2018.

[32] R. Amin, S. Knowlton, A. Hart et al., "3D-printed microfluidic devices," Biofabrication, vol. 8, no. 2, 2016.

[33] S. Knowlton, C. H. Yu, F. Ersoy, S. Emadi, A. Khademhosseini, and S. Tasoglu, "3D-printed microfluidic chips with patterned, cell-laden hydrogel constructs," Biofabrication, vol. 8, no. 2, article 025019, 2016.

[34] C. M. B. Ho, S. H. Ng, K. H. H. Li, and Y. J. Yoon, "3D printed microfluidics for biological applications," Lab on a Chip, vol. 15, no. 18, pp. 3627-3637, 2015.

[35] A. Dellaquila, Five short stories on the history of microfluidics, 2017.

[36] N.-T. Nguyen and S. Wereley, Fundamentals and Applications of Microfluidics, Artech, 2006.

[37] M. F. Hochella, "There's plenty of room at the bottom: nanoscience in geochemistry," Geochimica et Cosmochimica Acta, vol. 66, no. 5, pp. 735-743, 2002.

[38] R. P. Feynman, "There's plenty of room at the bottom [data storage]," Journal of Microelectromechanical Systems, vol. 1, no. 1, pp. 60-66, 1992.

[39] G. M. Whitesides, "The origins and the future of microfluidics," Nature, vol. 442, no. 7101, pp. 368-373, 2006.

[40] A. R. Abate, P. Mary, V. van Steijn, and D. A. Weitz, "Experimental validation of plugging during drop formation in a Tjunction," Lab on a Chip, vol. 12, no. 8, pp. 1516-1521, 2012.

[41] R.-L. Chien and W. J. Parce, "Multiport flow-control system for lab-on-a-chip microfluidic devices," Fresenius' Journal of Analytical Chemistry, vol. 371, no. 2, pp. 106-111, 2001.

[42] J.-H. Lue, Y. S. Su, and T. C. Kuo, "Workshop, cost-effective and streamlined fabrications of re-usable world-to-chip connectors for handling sample of limited volume and for assembling chip array," Sensors, vol. 18, no. 12, p. 4223, 2018.

[43] J. Zhong, Nanofluidics: a window into transport and phase change in nanoporous systems, [Ph.D. thesis], University of Toronto, Canada, 2019.

[44] A. Folch and M. Toner, "Cellular micropatterns on biocompatible materials," Biotechnology Progress, vol. 14, no. 3, pp. 388-392, 1998.

[45] L. Shang, Y. Cheng, and Y. Zhao, "Emerging droplet microfluidics," Chemical Reviews, vol. 117, no. 12, pp. 7964-8040, 2017.

[46] Y. Whulanza, D. S. Widyaratih, J. Istiyanto, and G. Kiswanto, "Realization and testing of lab-on-chip for human lung replication," ARPN Journal of Engineering and Applied Sciences, vol. 9, pp. 2064-2067, 2014.

[47] A. M. Ghaemmaghami, M. J. Hancock, H. Harrington, H. Kaji, and A. Khademhosseini, "Biomimetic tissues on a chip for drug discovery," Drug Discovery Today, vol. 17, no. 3-4, pp. 173-181, 2012.

[48] D. Huh, B. D. Matthews, A. Mammoto, M. Montoya-Zavala, H. Y. Hsin, and D. E. Ingber, "Reconstituting organ-level lung functions on a chip," Science, vol. 328, no. 5986, pp. 16621668, 2010.

[49] A. Williamson, S. Singh, U. Fernekorn, and A. Schober, "The future of the patient-specific body-on-a-chip," Lab on a Chip, vol. 13, no. 18, pp. 3471-3480, 2013.

[50] C. Moraes, J. M. Labuz, B. M. Leung, M. Inoue, T. H. Chun, and S. Takayama, "On being the right size: scaling effects in designing a human-on-a-chip," Integrative Biology, vol. 5, no. 9, pp. 1149-1161, 2013. 
[51] E. W. Esch, A. Bahinski, and D. Huh, "Organs-on-chips at the frontiers of drug discovery," Nature Reviews Drug Discovery, vol. 14, no. 4, pp. 248-260, 2015.

[52] R. Vitorino, S. Guedes, J. P. da Costa, and V. Kašička, "Microfluidics for peptidomics, proteomics, and cell analysis," Nanomaterials, vol. 11, no. 5, p. 1118, 2021.

[53] W. Lee, P. Tseng, and D. di Carlo, Microfluidic cell sorting and separation technology, 2017.

[54] D. Gao, C. Song, and J.-M. Lin, Microfluidics-mass spectrometry combination systems for single-cell analysis, 2019.

[55] R. Bogue, "MEMS sensors: past, present and future," Sensor Review, vol. 27, no. 1, pp. 7-13, 2007.

[56] J. W. Judy, "Microelectromechanical systems (MEMS): fabrication, design and applications," Smart Materials and Structures, vol. 10, no. 6, pp. 1115-1134, 2001.

[57] P. Pal and K. Sato, "Fabrication methods based on wet etching process for the realization of silicon MEMS structures with new shapes," Microsystem Technologies, vol. 16, no. 7, pp. 1165-1174, 2010.

[58] C. Iliescu and F. E. H. Tay, "Wet etching of glass," in CAS 2005 Proceedings. 2005 International Semiconductor Conference, 2005, Sinaia, Romania, October 2005.

[59] S. Ronggui and G. C. Righini, "Characterization of reactive ion etching of glass and its applications in integrated optics," Journal of Vacuum Science \& Technology A: Vacuum, Surfaces, and Films, vol. 9, no. 5, pp. 2709-2712, 1991.

[60] Hayashi Pure Chemical Ind, High performance anisotropic Si etchant in wet process, 2008.

[61] J. Albero, L. Nieradko, C. Gorecki et al., "Fabrication of spherical microlenses by a combination of isotropic wet etching of silicon and molding techniques," Optics Express, vol. 17, no. 8, pp. 6283-6292, 2009.

[62] M. Leester-Schädel, T. Lorenz, F. Jürgens, and C. Richter, "Fabrication of microfluidic devices," in Microsystems for Pharmatechnology, Springer International Publishing, 2016.

[63] J. J. Allen, Introduction to MEMS (MicroElectroMechanical Systems), 2007.

[64] V. K. Varadan, X. Jiang, and V. Varadan, Microstereolithography and other Fabrication Techniques for 3D MEMS, Wiley, 2001.

[65] M. J. Madou, Fundamentals of Microfabrication, CRC Press, 2018.

[66] D. Holbrook, "Controlling contamination: the origins of clean room technology," History and Technology, vol. 25, no. 3, pp. 173-191, 2009.

[67] J. Castillo-León, "Microfluidics and lab-on-a-chip devices: history and challenges," in Lab-on-a-Chip Devices and Micro-Total Analysis Systems, Springer International Publishing, 2015.

[68] W. J. Whitfield, Ultra-Clean Room, 1964.

[69] W. Whyte, "An introduction to the design of clean and containment areas," in Cleanroom DesignJohn Wiley \& Sons, Ltd.

[70] T. Sandle, “Application of quality risk management to set viable environmental monitoring frequencies in biotechnology processing and support areas," PDA Journal of Pharmaceutical Science and Technology, vol. 66, no. 6, pp. 560-579, 2012.

[71] W. Whyte, Cleanroom Technology: Fundamentals of Design, Testing and Operation, John Wiley \& Sons, 2010.
[72] Y. Lamkharbach, F. Bazi, L. Haji, L. Bennani, A. Mourran, and M. L. Bouamrani, "Study of changing statistics model's influence on the exploitation and conformity of results in the new standard version ISO 14644 part 1," Periodicals of Engineering and Natural Sciences (PEN), vol. 6, no. 2, 2018.

[73] D. Khorsandi, A. Fahimipour, P. Abasian et al., "3D and 4D printing in dentistry and maxillofacial surgery: printing techniques, materials, and applications," Acta Biomaterialia, vol. 122, pp. 26-49, 2021.

[74] A. K. Miri, E. Mostafavi, D. Khorsandi, S. K. Hu, M. Malpica, and A. Khademhosseini, "Bioprinters for organs-on-chips," Biofabrication, vol. 11, no. 4, p. 042002, 2019.

[75] S. N. Economidou and D. Douroumis, "3D printing as a transformative tool for microneedle systems: Recent advances, manufacturing considerations and market potential," Advanced Drug Delivery Reviews, vol. 173, pp. 60-69, 2021.

[76] J. R. Tumbleston, D. Shirvanyants, N. Ermoshkin et al., "Continuous liquid interface production of 3D objects," Science, vol. 347, no. 6228, pp. 1349-1352, 2015.

[77] J. C. Brooks, K. I. Ford, D. H. Holder, M. D. Holtan, and C. J. Easley, "Macro-to-micro interfacing to microfluidic channels using 3D-printed templates: application to time-resolved secretion sampling of endocrine tissue," The Analyst, vol. 141, no. 20, pp. 5714-5721, 2016.

[78] M. P. de Beer, H. L. van der Laan, M. A. Cole, R. J. Whelan, M. A. Burns, and T. F. Scott, "Rapid, continuous additive manufacturing by volumetric polymerization inhibition patterning," Science Advances, vol. 5, no. 1, p. eaau8723, 2019.

[79] B. E. Kelly, I. Bhattacharya, H. Heidari, M. Shusteff, C. M. Spadaccini, and H. K. Taylor, "Volumetric additive manufacturing via tomographic reconstruction," Science, vol. 363, no. 6431, pp. 1075-1079, 2019.

[80] A. V. Nielsen, M. J. Beauchamp, G. P. Nordin, and A. T. Woolley, "3D printed microfluidics," Annual Review of Analytical Chemistry, vol. 13, no. 1, pp. 45-65, 2020.

[81] C. M. Spadaccini, G. Farquar, T. Weisgraber et al., High resolution projection micro stereolithography system and method., 2016.

[82] D. Khorsandi, S. Palacios, Y. Gaslain et al., "P159 Human uterine cervix-on-a-chip: establishing the first in vitro model to study the development of cervical carcinoma and human papiloma virus mechanism of action," in Poster Exhibition Day 2, BMJ Publishing Group Ltd, 2019.

[83] K. C. Hribar, P. Soman, J. Warner, P. Chung, and S. Chen, "Light-assisted direct-write of 3D functional biomaterials," Lab on a Chip, vol. 14, no. 2, pp. 268-275, 2014.

[84] K. A. Homan, D. B. Kolesky, M. A. Skylar-Scott et al., "Bioprinting of 3D convoluted renal proximal tubules on Perfusable chips," Scientific Reports, vol. 6, no. 1, 2016.

[85] S. Wu, J. Serbin, and M. Gu, "Two-photon polymerisation for three-dimensional micro-fabrication," Journal of Photochemistry and Photobiology A: Chemistry, vol. 181, no. 1, pp. 1-11, 2006.

[86] H. Petek, M. J. Weida, H. Nagano, and S. Ogawa, "Real-time observation of adsorbate atom motion above a metal surface," Science, vol. 288, no. 5470, pp. 1402-1404, 2000.

[87] S. Maruo, K. Ikuta, and H. Korogi, "Force-controllable, optically driven micromachines fabricated by single-step twophoton microstereolithography," Journal of Microelectromechanical Systems, vol. 12, no. 5, pp. 533-539, 2003. 
[88] F. Abe, "The manufacturing of hard tools from metallic powders by selective laser melting," Journal of Materials Processing Technology, vol. 111, no. 1-3, pp. 210-213, 2001.

[89] H. Exner and A. Streek, High resolution laser micro sintering / melting using q-switched and high brilliant laser radiation, $\mathrm{H}$. Helvajian, A. Piqué, M. Wegener, and B. Gu, Eds., 2015.

[90] T. Petsch, P. Regenfuss, R. Ebert et al., "Industrial laser micro sintering," in International Congress on Applications of Lasers \& Electro-Optics, Laser Institute of America, 2004.

[91] J. Bohandy, B. F. Kim, and F. J. Adrian, "Metal deposition from a supported metal film using an excimer laser," Journal of Applied Physics, vol. 60, no. 4, pp. 1538-1539, 1986.

[92] S. Papazoglou and I. Zergioti, "Laser induced forward transfer (LIFT) of nano-micro patterns for sensor applications," Microelectronic Engineering, vol. 182, pp. 25-34, 2017.

[93] W. A. Tolbert, I.-Y. S. Lee, M. M. Doxtader, E. W. Ellis, and D. D. Dlott, "High-speed color imaging by laser ablation transfer with a dynamic release layer: fundamental mechanisms," Journal of Imaging Science, vol. 37, pp. 411-421, 1993.

[94] D. B. Chrisey, A. Pique, J. Fitz-Gerald et al., "New approach to laser direct writing active and passive mesoscopic circuit elements," Applied Surface Science, vol. 154-155, pp. 593600, 2000.

[95] A. Piqué and B. C. Douglas, Direct-Write Technologies for Rapid Prototyping Applications: Sensors, Electronics, and Integrated Power Sources, 2001.

[96] C. W. Visser, R. Pohl, C. Sun, G. W. Römer, B. Huis in 't Veld, and D. Lohse, "Toward 3D printing of pure metals by laserinduced forward transfer," Advanced Materials, vol. 27, no. 27, pp. 4087-4092, 2015.

[97] H. Alemohammad and E. Toyserkani, "Laser-assisted additive fabrication of micro-sized coatings," in Advances in Laser Materials Processing, Elsevier, 2010.

[98] A. Piqué, H. Kim, R. C. Y. Auyeung, and A. T. Smith, "Laser forward transfer of functional materials for digital fabrication of microelectronics," Journal of Imaging Science and Technology, vol. 57, no. 4, pp. 1-8, 2013.

[99] A. J. Birnbaum, H. Kim, N. A. Charipar, and A. Piqué, "Laser printing of multi-layered polymer/metal heterostructures for electronic and MEMS devices," Applied Physics A, vol. 99, no. 4, pp. 711-716, 2010.

[100] R. C. Y. Auyeung, H. Kim, A. J. Birnbaum, M. Zalalutdinov, S. A. Mathews, and A. Piqué, "Laser decal transfer of freestanding microcantilevers and microbridges," Applied Physics A, vol. 97, no. 3, pp. 513-519, 2009.

[101] C. Duty, D. Jean, and W. J. Lackey, "Laser chemical vapour deposition: materials, modelling, and process control," International Materials Reviews, vol. 46, no. 6, pp. 271-287, 2001.

[102] M. C. Wanke, O. Lehmann, K. Muller, Q. Wen, and M. Stuke, "Laser rapid prototyping of photonic band-gap microstructures," Science, vol. 275, no. 5304, pp. 1284-1286, 1997.

[103] I. Gibson, D. Rosen, and B. Stucker, "Additive manufacturing technologies: 3D printing, rapid prototyping, and direct digital manufacturing, second edition," in Additive Manufacturing Technologies: 3D Printing, Rapid Prototyping, and Direct Digital Manufacturing, Second Edition, pp. 1-498, 2015.

[104] J. Lessing, A. C. Glavan, S. B. Walker, C. Keplinger, J. A. Lewis, and G. M. Whitesides, "Inkjet printing of conductive inks with high lateral resolution on omniphobic " $\mathrm{R}{ }^{\mathrm{F}}$ paper" for paper-based electronics and MEMS," Advanced Materials, vol. 26, no. 27, pp. 4677-4682, 2014.

[105] V. Tagliaferri, F. Trovalusci, S. Guarino, and S. Venettacci, "Environmental and economic analysis of FDM, SLS and MJF Additive Manufacturing Technologies," Materials, vol. 12, no. 24, p. 4161, 2019.

[106] J. A. Wittkopf, K. Erickson, P. Olumbummo, A. Hartman, H. Tom, and L. Zhao, "3D printed electronics with multi jet fusion," NIP \& Digital Fabrication Conference, vol. 2019, no. 1, pp. 29-33, 2019.

[107] A. C. Lamont, A. T. Alsharhan, and R. D. Sochol, "Geometric Determinants of In-Situ Direct Laser Writing," Scientific Reports, vol. 9, no. 1, p. 394, 2019. 